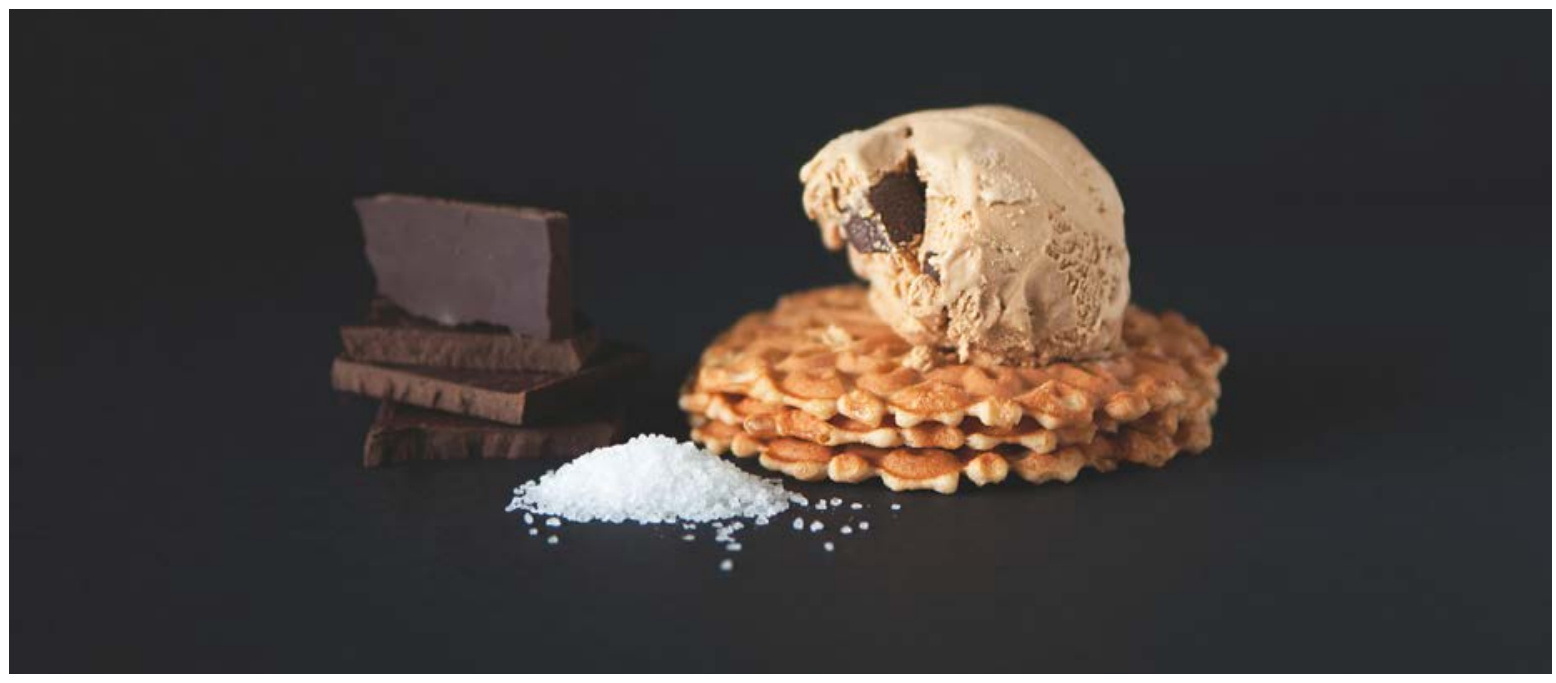

by Franco Bagnoli,

Dept. of Physics and Astronomy and Center for the Study of Complex Dynamics - University of Florence, Italy franco.bagnoli@unifi.it - DOI: http://dx.doi.org/10.1051/epn/2016204

\title{
Delicious ice cream why does salt thaw ice?
}

During winter, we use to spread salt to thaw ice on the streets. In a physics show, one can be almost sure that after showing this effect, the answer to what happens to temperature will be "it increases". But no! It goes down, in such amount that one can complement the show by producing hand-made ice creams [1].

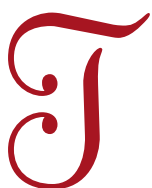

his phenomenon is also exploited for self-injuring [2] (unbelievers may be asked to hold a salted ice cube in their hand). Clearly, publicly producing almost-instant ice cream with kitchen equipment (i.e., without liquid nitrogen) is an easy way of getting attendees' attention.

This phenomenon is not so easy to explain because one cannot simply rely on energy considerations. Indeed, water molecules and salt ions (sodium and chlorine) prefer to stay separate from an energy point of view and so they do, below $-21^{\circ} \mathrm{C}{ }^{1}$

As can be seen in Figure 1, the chlorine and sodium ions (being charged) fit well among water molecules which are

1 Therefore, it is useless to spread salt on street ice below such temperature.

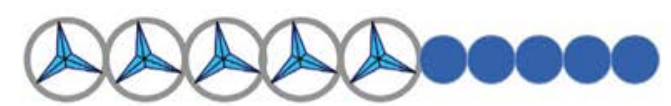

Separated ice and salt: 1 configuration

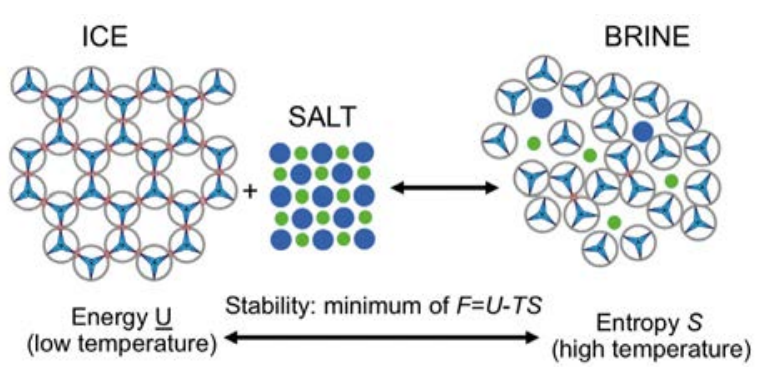

$\triangle$ FIG.1: A schematic representation of the mixture of water and salt ions using the Mercedes-Benz model.

polar, but by doing so they disrupt the ordered structure of ice and salt crystals. As for melting, this implies that the mixture is stable at high temperatures, and that the separate crystals are stable at low temperatures, below $-21^{\circ} \mathrm{C}$. So why

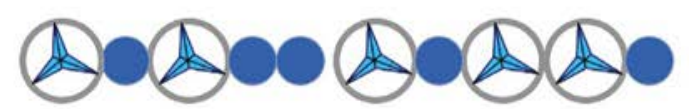

Mixed ice and salt: many configurations

\FIG.2: Number of configuration in ordered and disordered arrangements of molecules and ions in a one-dimensional model. 


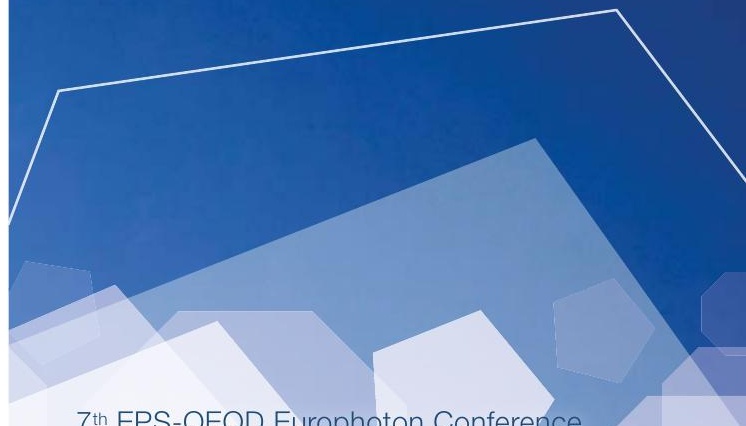

does the temperature go down when salt is mixed with ice?

Let us come back to the previous statement: disordered structures are stable at high temperatures, and ordered ones at low temperatures. The latter is clearly favoured by energy, but the first? We have to introduce the concept of entropy $S$, which is the (logarithm of) the number of possible configurations.

Using a simple one-dimensional model (Fig. 2), we can appreciate the difference in the number of configurations for ordered and disordered structures. If the difference in energy between the two types of structures is large, the order is preferred with occasional and local fluctuations (for temperatures above the absolute zero and in a classical framework). But if the difference in energy is not so large, fluctuations that raise energy happen more frequently. And when order is destroyed, it is recovered with difficulty because there are so many un-ordered configurations nearby, not so distant in energy, and only one ordered.

Let us invoke the concept of free energy $F=U-T S$, where $U$ is the energy and $T$ the temperature. The stability of a system is given by a minimum of $F$, and from the above formula it is easy to see that the temperature favours the energy when it is low, and the entropy (that has a minus sign) when it is large.

So, when salt is added to ice above - 21 degrees, molecules tend to reach a stable configuration by mixing, but this requires energy in order to break the hydrogen bonds of ice and for the dissolution of the salt (the latent heat of ice is $6.01 \mathrm{~kJ} / \mathrm{mol}$ and the dissolution of sodium chloride requires $3.87 \mathrm{~kJ} / \mathrm{mol}$ ).

And now our challenge (for people using the International System of Units): why is the Fahrenheit scale so weird and why does its zero correspond to (roughly) -18 degrees? ${ }^{2}$

\section{References}

[1] Steve Spangler, The Spangler Effect - Homemade Ice Cream, https: //www.youtube.com/watch?v=Y5XzhcDq5Bw.

[2] Salt and ice challenge, http://en.wikipedia.org/wiki/Salt_and_ice_ challenge.

[3] The Fahrenheit scale http://en.wikipedia.org/wiki/Fahrenheit.

${ }^{2}$ At the time of Daniel Gabriel Fahrenheit (1686-1736), the mixture of salt, ice and water constituted the lowest temperature easily obtainable without laboratory tools, and was used to define the zero of the Fahrenheit scale (using equal volumes of ice and salt), while 100 degrees corresponded (roughly) to the body temperature [3]. The advantages of this scale is that it never requires negative temperatures in everyday life, and (with the present refined scale) that there is a 180 degree separation between the boiling and freezing points of water, making easier to measure temperatures without decimals.

\section{1-26 August 2016}

\section{Vienna, Austria www.europhoton.org}

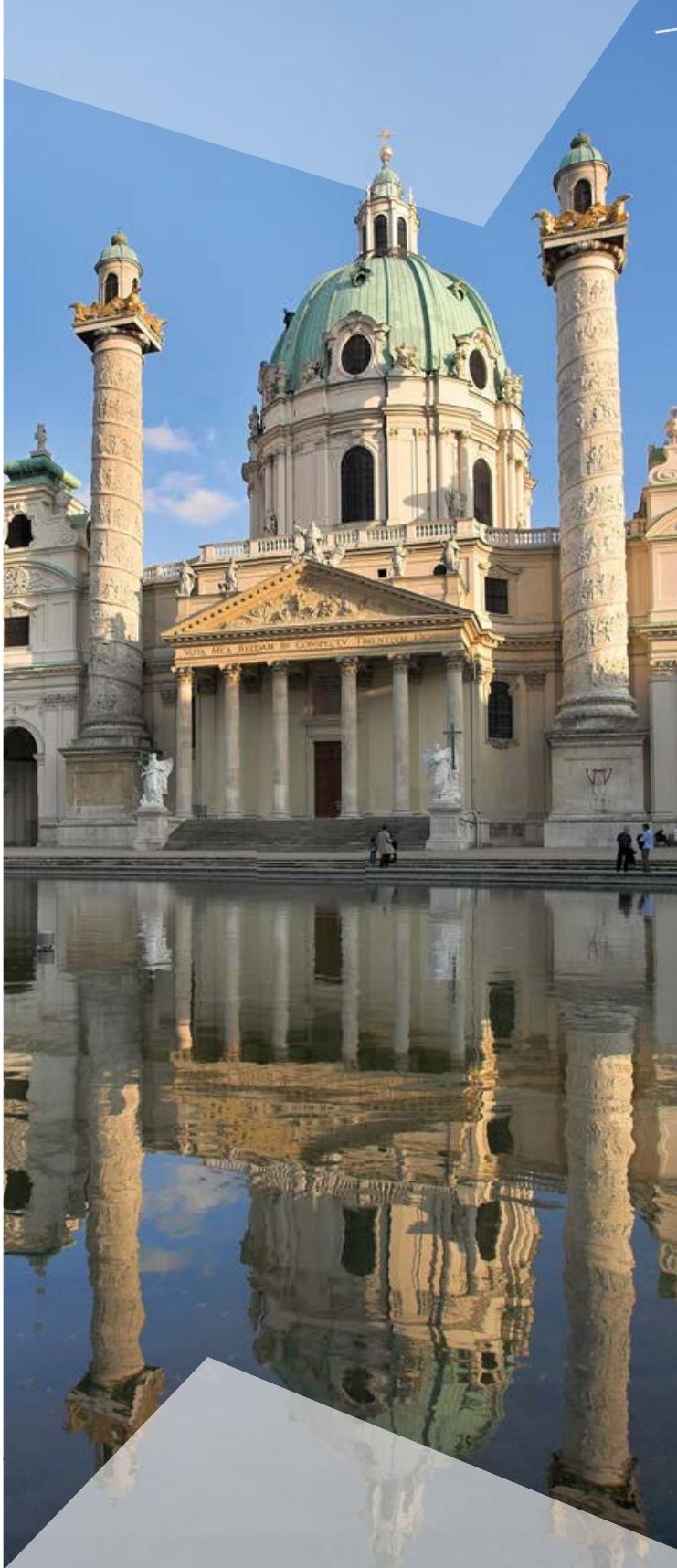

Abstract Submission Deadline Thursday, 21 ${ }^{\text {st }}$ April 2016 